\title{
PANUVEÍTIS BILATERAL SIFILÍTICA: A PROPÓSITO DE UN CASO
}

\author{
SYPHILITIC BILATERAL PANUVEITIS: A CASE REPORT
}

\author{
PIÑÓN-MOSQUERA R ${ }^{1}$, JIMÉNEZ-BENITO J², OLEA-CASCÓN J²
}

\begin{abstract}
RESUMEN
Caso clínico: Mujer de 57 años inmunocompetente es diagnosticada de panuveítis bilateral sifilítica tras presentar un cuadro dramático de déficit visual severo y bilateral con inflamación del segmento anterior, importante vitritis y coriorretinitis que se resuelven favorablemente tras el tratamiento.

Discusión: La sífilis es una enfermedad cuya frecuencia está en aumento. A nivel ocular puede provocar pérdida severa de visión si no se diagnostica a tiempo ya que puede originar neuritis y coriorretinitis con panuveítis, siendo el 50\% de ellas bilaterales. Sin embargo, se considera una de las pocas causas de uveítis que con tratamiento específico se pueden curar.
\end{abstract}

Palabras clave: Sífilis, panuveítis bilateral, coriorretinitis, ceguera, tratamiento.

\begin{abstract}
Case report: A 57-year-old immunocompetent woman was diagnosed with syphilitic bilateral panuveitis after the onset of dramatic bilateral visual loss with inflammation of the anterior segment of the eye, severe vitritis and chorioretinitis which improved after treatment.

Discussion: Syphilis is a complex disease of increasing prevalence. If diagnosis is delayed ocular syphilis can produce severe visual loss due to neuritis, chorioretinitis with panuveitis, affecting both eyes in $50 \%$ of cases. Nevertheless, syphilis is considered one of the few causes of uveitis in which a cure can be obtained with proper treatment (Arch Soc Esp Oftalmol 2009; 84: 101-104).
\end{abstract}

Key words: Syphilis, bilateral panuveitis, chorioretinitis, blindness, treatment.

\section{INTRODUCCIÓN}

La sífilis es una infección de transmisión sexual conocida desde hace siglos habiéndose descrito una primera epidemia a finales del siglo XV. Su agente causal, el Treponema pallidum, se descubrió en 1905 por Schaudinn y Hoffman. En 1918 Ygersheimer describió «la enfermedad ocular sifilítica». En
1943, la introducción de la penicilina revolucionó el tratamiento de esta enfermedad (1).

La afectación ocular puede ocurrir tanto en la sífilis congénita como en la adquirida afectando a cualquier estructura del ojo $(1,2)$.

La incidencia de uveítis sifilítica en pacientes con sífilis varía entre el 4 y el 14\% según las series y actualmente está aumentando en relación al resto de uveítis.

\footnotetext{
Recibido: 14/4/07. Aceptado: 9/2/09.

Complejo Asistencial de Burgos. Servicio de Oftalmología. Burgos. España.

1 Doctor en Medicina.

2 Licenciado en Medicina.

La institución es el lugar de trabajo, no teniendo ninguna responsabilidad de lo escrito en este artículo.

Correspondencia:

Rosa Piñón Mosquera

C/. Francia, 24

09006 Burgos

España

E-mail: pozopinon@telefonica.net
} 
Las características de las iridociclitis y coriorretinitis sifilíticas no son patognomónicas. La coriorretinitis puede ser difusa, multifocal y profunda. Los signos precoces incluyen vitritis, coriorretinitis multifocal, neuritis óptica, vasculitis, hemorragias y edema retiniano.

La pigmentación se presenta tardíamente. Se describen como panuveítis, casos de uveítis con visión borrosa, dolor, enrojecimiento, fotofobia, manchas flotantes uni o bilaterales, agudas o crónicas.

\section{CASO CLÍNICO}

Mujer de 57 años, inmunocompetente que acudió a urgencias por molestias oculares y alteración de

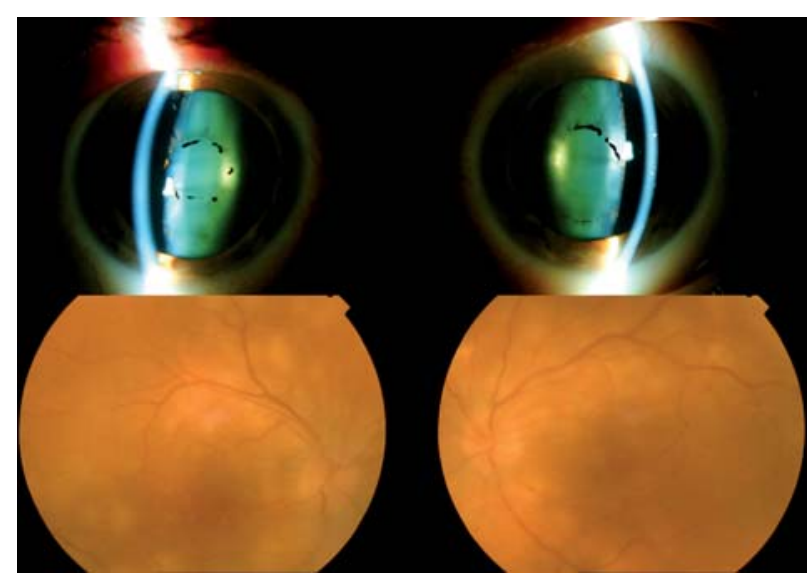

Fig. 1: Imagen del segmento anterior tras romper sinequias y F.O. visión bilateral. En la exploración la agudeza visual (AV) era de 0,125 en el ojo derecho (OD) y 0,2 en el ojo izquierdo (OI). En la Biomicroscopía se observaba una queratitis con pliegues endoteliales y sinequias posteriores en ambos ojos (AO), células de $1+$ de intensidad en cámara anterior (CA). La tensión ocular era normal en AO. En fondo de ojo (FO) se observaba vitritis bilateral intensa, sospechando la presencia de vasculitis . Se pautó tratamiento tópico con colirios de dexametasona, trobramicina y atropina.

A las 48 horas la visión disminuyó a movimiento de manos en $\mathrm{AO}$ con vitritis más intensa. Se decidió ingreso hospitalario y tratamiento con dexametasona tópica (pauta de las 90 gotas), metilprednisolona (40 mg cada $12 \mathrm{~h}$ ) y Ciprofloxacino (200 mg cada $12 \mathrm{~h}$ ) intravenosos.

Una ligera mejoría, a los 7 días, en la inflamación del polo anterior y la rotura de sinequias permitió ver mejor el FO observando la turbidez vítrea y focos de coriorretinitis en polo posterior (fig. 1).

La serología infecciosa fue positiva para la sífilis, TPHA + (1/320) sin otros hallazgos analíticos. Las pruebas de imagen, resonancia magnética nuclear de cráneo y órbitas y la radiografía de tórax no presentaban alteraciones.

En la anamnesis más detallada la paciente informa de una infección ginecológica que pudiera ser compatible con chancro sifilítico hace 35 años.

Por la posibilidad de sífilis neurológica se decide punción de LCR que informan como normal.

Tras repetir la serología, siendo el TPHA + $(1 / 640)$ con Ac Totales + para Lues, se decidió tra-

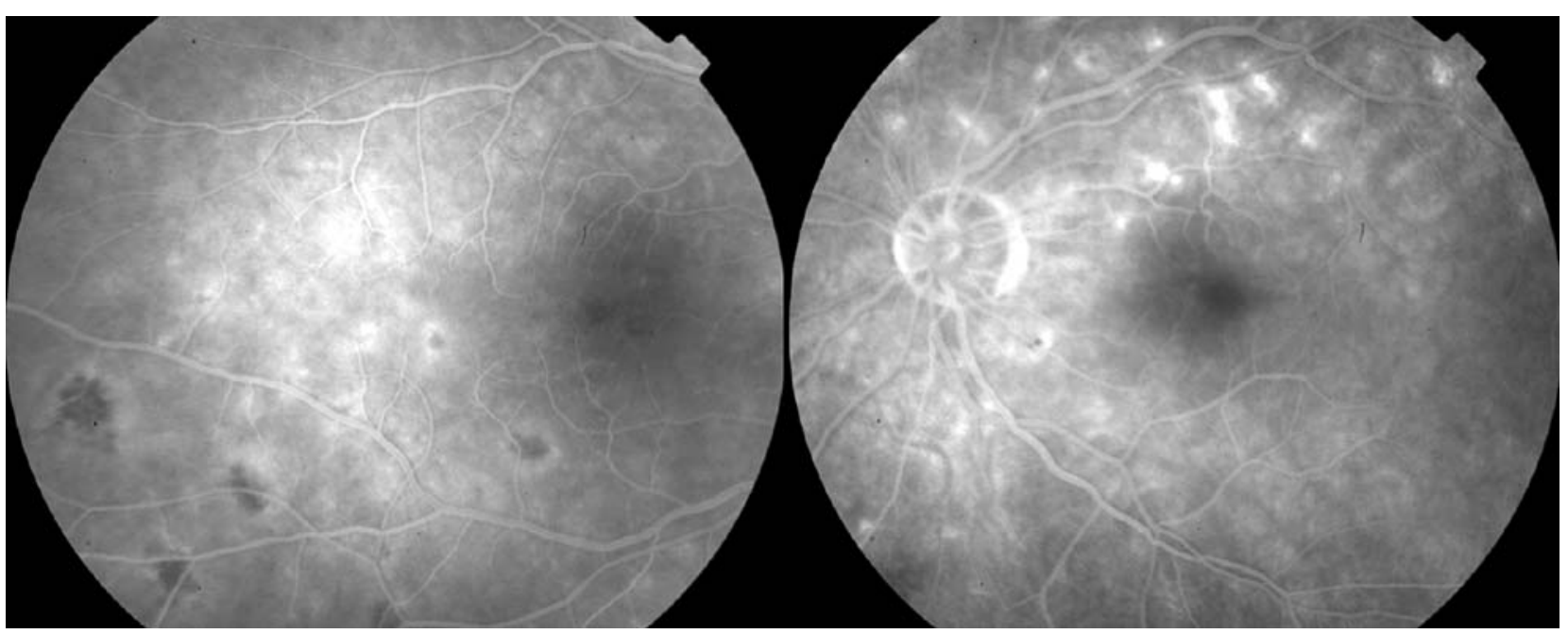

Fig. 2: Focos de coriorretinitis con hiperfluorescencia en AFG en ambos ojos. 


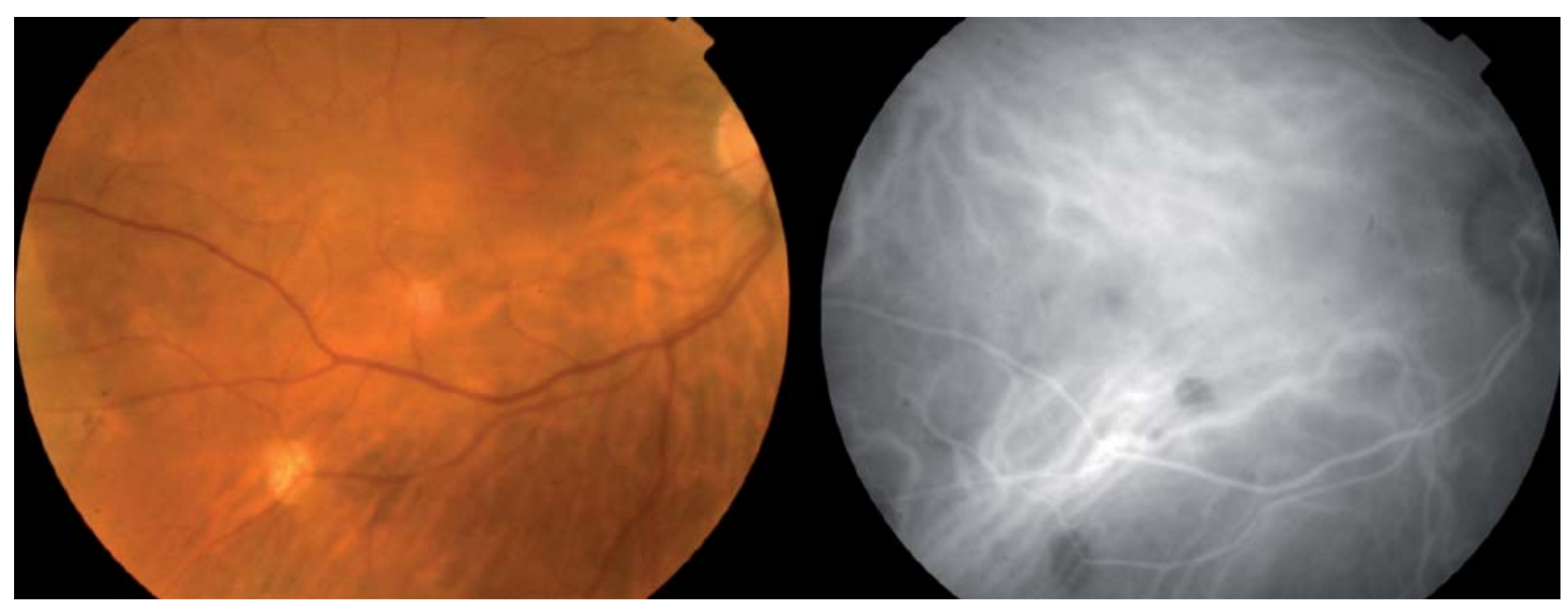

Fig. 3: Imagen de F.O. y Angiografía con Verde Indocianina con áreas hipofluorescentes en OD.

tar con Penicilina G Benzatina 2,4 millones de U.I. intramusculares cada semana durante 3 semanas, además de seguir con corticoides tópicos y generales en pauta descendente.

La evolución clínica fue favorable, mejorando la agudeza visual a 0,1 en OD y 0,3 en OI después de la primera inyección. La AV continuó mejorando aunque con importante afectación del campo visual (CV) con disminución de la sensibilidad generalizada y bilateral.

En revisiones posteriores se observaban mejor los focos de coriorretinitis, con áreas hiperfluorescentes en tiempos precoces en la angiografía fluoresceínica (AFG), que se mantienen en tiempos tardíos y se corresponden con hipofluorescencia con verde indocianina (ICG) en fases intermedias y tardías en relación con posibles granulomas coroideos, que posteriormente van a ir cicatrizando dejando una atrofia del epitelio pigmentario (figs. 2 y 3 ).

A los 3 meses la AV en OD era 0,8 y unidad en OI manteniéndose igual a los 6, 12 y 24 meses de evolución, con focos de atrofia coriorretinianos dispersos sin signos de actividad en vítreo y con un CV normal en $\mathrm{AO}$ (fig. 4).

\section{DISCUSIÓN}

La sífilis es conocida como una de las «grandes simuladoras» en medicina, su incidencia está

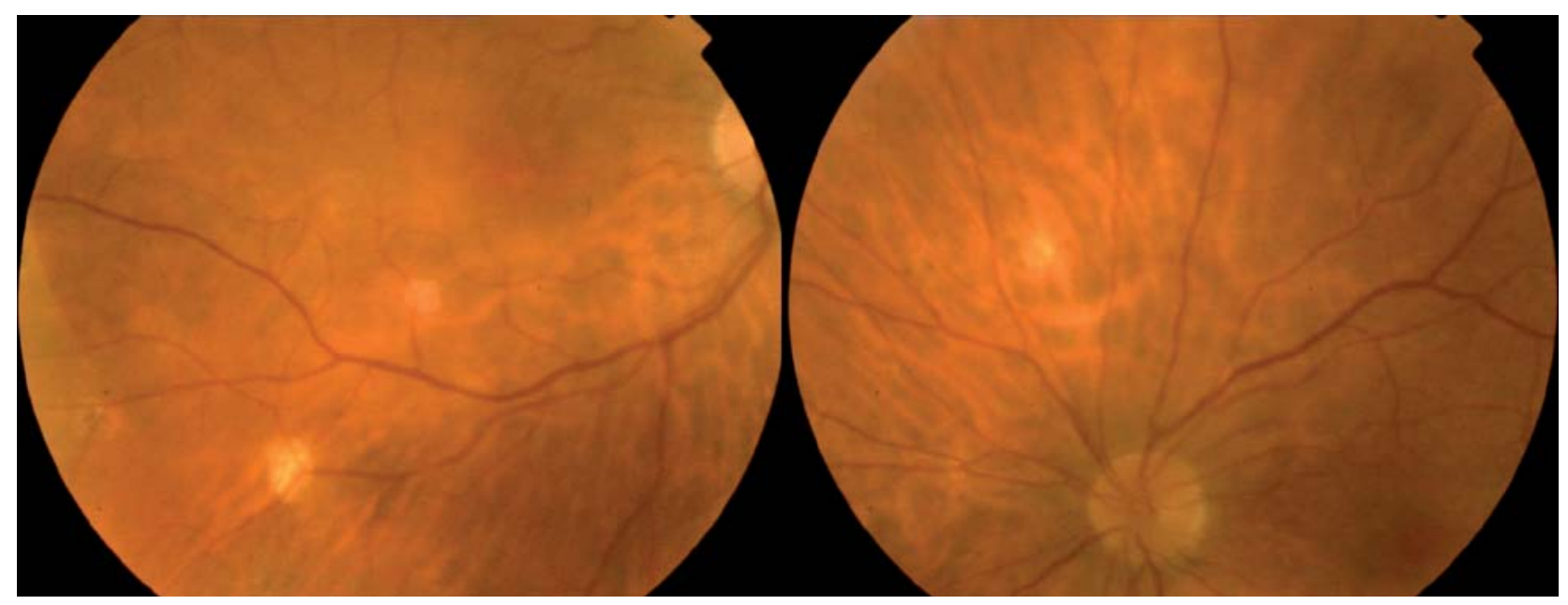

Fig. 4: Imagen de F.O. de ambos ojos a los 24 meses de evolución. 
aumentando $(3,4)$ y debe considerarse como una etiología posible en toda uveítis.

La manifestación más frecuente es una coriorretinitis focal o generalizada, que al cicatrizar simula una retinitis pigmentaria atípica y hasta en un $50 \%$ de casos es bilateral.

En la AFG se pueden ver focos de hiperfluorescencia progresiva, siendo un signo típico la «arteritis». En la ICG si existe una infiltración inflamatoria de todo el espesor coroideo se dificulta la fluorescencia hasta tiempos tardíos como en el caso que nos ocupa.

El diagnóstico viene dado por la positividad de test serológicos específicos y clínica compatible. Si la serología es positiva debe descartarse neurosífilis mediante punción lumbar y estudio de los marcadores serológicos pertinentes.

El diagnóstico diferencial de la panuveítis sifilítica incluye Tuberculosis, Enfermedad de Lyme, Sarcoidosis y Enfermedad de Vogt-Koyanagi-Harada entre otras.

Siempre que estemos ante una afectación ocular por sífilis es obligado hacer serología VIH, que en esta paciente resultó ser negativa.
Una vez establecido el diagnóstico de coriorretinitis sifilítica se tratará con penicilina a la dosis ya mencionada, la cual sería mayor si el LCR fuera positivo. En casos de alergia a penicilina se indicaría eritromicina o tetraciclinas $(4,5)$.

\section{BIBLIOGRAFÍA}

1. Weinberg R. Syphilis. In: Duanes's Clinical Ophthalmology. Philadelphia: Lippincott Williams \& Wilkins; 2000; IV: Chapter 50: 1-5.

2. Kiss S, Damico FM, Young LH. Ocular manifestations and treatment of syphilis. Semin Ophthalmol 2005; 20: 161167.

3. Doris JP, Saha K, Jones NP, Sukthankar A. Ocular syphilis: the new epidemic. Eye 2006; 20: 703-705.

4. Kasper DL, Braunwald E, Fauci AS, Hauser SL, Longo DL, Jameson JL. Enfermedades de transmisión sexual e infecciones del aparato reproductor. In: Harrison Manual de Medicina. Madrid: McGraw-Hill-Interamericana de España, S.A.U; 2005; 422-425.

5. Opremcak EM, Cunningham ET, Foster CS, Forster D, Moorthy RS, Lopatynsky M. Intraocular inflammation and uveitis, In: Liesengang TJ, Skuta GL, Cantor LB. Basic and Clinical Sciences Courses. San Francisco: AAO; 2005-2006; 9: 187-193. 